\title{
Effect of in vitro fertilization medium on the acrosome reaction, cortical reaction, zona pellucida hardening and in vitro development in pigs
}

\author{
P. Coy, J. Gadea, R. Romar, C. Matás and E. García \\ Departamento de Fisiología, Facultad de Veterinaria, Universidad de Murcia, \\ 30071 Murcia, Spain
}

Physiological events at the time of fertilization of pig oocytes may differ in vitro depending on the in vitro fertilization (IVF) medium. This hypothesis was tested by in vitro maturation of pig oocytes for $44 \mathrm{~h}$ in NCSU-37 medium and thereafter fertilization with frozen-thawed ejaculated spermatozoa. Three different IVF media (TCM-199, Tyrode's albumin lactate pyruvate (TALP) and Tris-buffered medium (TBM)) were used. For the acrosome reaction test, spermatozoa were incubated for 0-150 $\mathrm{min}$ in the three IVF media, and the proportion of live acrosome-reacted and acrosome-intact cells was determined by fluorescein isothiocyanate-labelled peanut agglutinin (FITC-PNA) and propidium iodide (PI) staining. The cortical granule density of oocytes was evaluated by confocal microscopy, 2.5 and $5.0 \mathrm{~h}$ after culture in each medium in the presence or absence of spermatozoa. Zona pellucida resistance to pronase digestion was also determined in the same groups. The percentages of penetration, monospermy, male pronucleus formation, cleavage and blastocyst formation, and the number of cells per blastocyst after culture were determined. The results indicate that the acrosome reaction occurred much faster in TBM than in TCM-199 or TALP medium. Continuous cortical granule synthesis was observed in the three media when oocytes were incubated in the absence of spermatozoa. The presence of spermatozoa triggered the cortical reaction in a large proportion of oocytes fertilized in TCM-199 and TALP media. On the basis of the duration of pronase digestion, the zona pellucida of oocytes incubated in TCM-199 was harder $(407.7 \pm 35.5 \mathrm{~s})$ than that of oocytes cultured in TALP $(235.4 \pm 18.2 \mathrm{~s})$ or TBM (189.1 $\pm 16.8 \mathrm{~s})$. No zona pellucida hardening was noted in oocytes after insemination in any of the media. The percentages of penetration and cleavage were higher in oocytes cultured in TCM-199 and TALP than in TBM. The percentage of monospermy was higher in TCM-199 and TBM than in TALP. No effect of the medium was shown on the percentage of blastocyst formation or on the number of cells per blastocyst. In conclusion, the results highlight how differently the fertilization events take place in each IVF medium and how far these IVF media still are from achieving biological properties of gametes close to those observed in the physiological setting.

\section{Introduction}

At the time of fertilization under physiological conditions, a particular pattern of activation events occurs in a speciesspecific temporal progression (Ducibella, 1998). Thus, by sperm-egg interaction, one capacitated spermatozoon binds to the zona pellucida (ZP) and undergoes the acrosome reaction before crossing the perivitelline space to reach the plasma membrane of the oocyte. This last binding to the plasma membrane triggers cell cycle resumption and cortical granule exocytosis causing changes in the ZP that prevent polyspermic penetration. All these events are closely linked to a transient increase in intracellular free $\mathrm{Ca}^{2+}$ concentration followed by a series of periodic $\mathrm{Ca}^{2+}$ waves due to release of $\mathrm{Ca}^{2+}$ from intracellular storage (Fissore et al., 1992; Abbott and Ducibella, 2001). The

Email:pcoy@um.es period in which these events occur, although much longer in mammals than in echinoderms, may be as short as the first $3 \mathrm{~h}$ of contact. Under in vitro conditions, the pattern of each of these events does not reflect the situation in vivo. The fluid in which in vitro fertilization (IVF) takes place is quite different from oviductal fluid, and there is no single medium commonly used by researchers to reproduce fertilization conditions. Calcium availability in the medium and its subsequent uptake by the oocyte or spermatozoon may have potential effects on intracellular $\mathrm{Ca}^{2+}$ concentrations modifying these events and indeed, early studies in pigs identified the $\mathrm{Ca}^{2+}$ concentration of the IVF medium as a key factor (Cheng, 1985).

For IVF in pigs, even when the $\mathrm{Ca}^{2+}$ concentration in the medium is high, abnormal fertilization, mainly due to polyspermy, is a frequent event and many studies have addressed this problem (for a review, see Coy and Romar, in press). In contrast, few studies have explored the biological variables, such as the acrosome reaction, cortical granule 
exocytosis or ZP hardening under similar conditions, that contribute towards the success of the technique. This type of investigation may improve understanding of the cause of polyspermy or any other factor that reduces IVF efficiency.

The hypothesis tested in the present study was that these physiological events probably show different behaviour patterns when the gametes are cultured in different IVF media. The birth of piglets after IVF has been achieved on several occasions mainly through the use of the IVF media: TCM-199, Tyrode's albumin lactate pyruvate (TALP) and Tris-buffered medium (TBM) (Cheng, 1985; Mattioli et al., 1989; Yoshida et al., 1993a; Abeydeera et al., 1998; Rath et al., 1999). On the basis of these results, the aim of the present study was to evaluate how TCM-199, TALP and TBM affect the acrosome reaction pattern, cortical granule density and distribution, ZP hardening, sperm penetration and early embryonic development in pig gametes under the same experimental conditions. As suggested by Wang et al. (1997), knowledge of these effects would improve current understanding of the IVF process.

\section{Materials and Methods}

\section{Oocyte collection and in vitro maturation}

Unless otherwise indicated, all the chemicals used in this study were purchased from Sigma-Aldrich Química SA (Madrid). Ovaries from prepubertal gilts were transported to the laboratory within $30 \mathrm{~min}$ after slaughter in saline $(0.9 \%$ (w/v) $\mathrm{NaCl}$ ) containing $100 \mathrm{mg}$ kanamycin $\mathrm{ml}^{-1}$ at $37^{\circ} \mathrm{C}$. Cumulus-oocyte complexes (COCs) were collected from non-atretic follicles (3-6 mm in diameter) by slicing, and were washed twice in modified Dulbecco's PBS supplemented with $1 \mathrm{mg}$ polyvinyl alcohol $\mathrm{ml}^{-1}$. Only oocytes with a homogeneous cytoplasm and a complete and dense cumulus oophorus were matured. The selected complexes were then washed again twice in maturation medium that had been equilibrated for a minimum of $3 \mathrm{~h}$ at $38.5^{\circ} \mathrm{C}$ under $5 \% \mathrm{CO}_{2}$ in air.

The medium used for oocyte maturation was NCSU-37 (Petters and Wells, 1993) supplemented with $0.57 \mathrm{mmol}$ cysteine $\mathrm{I}^{-1}$, 1 mmol dibutyryl cAMP I-1, $5 \mu \mathrm{g}$ insulin $\mathrm{ml}^{-1}$, $50 \mu \mathrm{mol} \beta$-mercaptoethanol I-1, 10 iu eCG ml-1 (Folligon; Intervet International BV, Boxmeer), 10 iu hCG ml-1 (Chorulon; Intervet International BV, Boxmeer), and 10\% $(\mathrm{v} / \mathrm{v})$ pig follicular fluid obtained from only one pool of follicles 3-6 mm in diameter and stored at $-20^{\circ} \mathrm{C}$ until use.

Groups of 50 oocytes were cultured into each well of a Nunc four-well multi-dish containing $500 \mu \mathrm{l}$ maturation medium for $22 \mathrm{~h}$ at $38.5^{\circ} \mathrm{C}$ under $5 \% \mathrm{CO}_{2}$ in air. After culture, oocytes were washed three times and transferred to hormone-free maturation medium for a further $22 \mathrm{~h}$ (Funahashi and Day, 1993).

\section{In vitro fertilization}

At the end of the maturation period, groups of 50 oocytes were transferred to $2 \mathrm{ml}$ of one of three fresh IVF media
Table 1. Composition of media used for IVF in pigs

\begin{tabular}{|c|c|c|c|}
\hline $\begin{array}{l}\text { Concentration } \\
\left(\mathrm{mmol} \mathrm{I}^{-1}\right)\end{array}$ & TCM-199a & TALP & TBM \\
\hline $\mathrm{NaCl}$ & 116.35 & 114.06 & 113.10 \\
\hline $\mathrm{KCl}$ & 5.36 & 3.20 & 3.00 \\
\hline $\mathrm{CaCl}_{2} \cdot 2 \mathrm{H}_{2} \mathrm{O}$ & 1.80 & - & 7.50 \\
\hline Ca-Lactate $5 \mathrm{H}_{2} \mathrm{O}$ & 8.75 & 8.00 & - \\
\hline $\mathrm{MgCl}_{2} \cdot 6 \mathrm{H}_{2} \mathrm{O}$ & - & 0.50 & - \\
\hline $\mathrm{MgSO}_{4}$ & 0.81 & - & - \\
\hline $\mathrm{NaH}_{2} \mathrm{PO}_{4}$ & 1.01 & 0.35 & - \\
\hline $\mathrm{NaHCO}_{3}$ & 25.07 & 25.07 & - \\
\hline Sodium lactate $\left(\mathrm{ml} \mathrm{I}^{-1}\right)$ & & 10.00 & - \\
\hline Sodium pyruvate & 0.90 & 1.10 & 5.00 \\
\hline Glucose & 3.05 & 5.00 & 11.00 \\
\hline Caffeine & 3.60 & 2.00 & 1.00 \\
\hline Tris & & & 20.00 \\
\hline $\mathrm{BSA}\left(\mathrm{mg} \mathrm{ml}^{-1}\right)^{\mathrm{b}}$ & - & 3.00 & 1.00 \\
\hline $\mathrm{PVA}\left(\mathrm{mg} \mathrm{ml}^{-1}\right)$ & & 1.00 & - \\
\hline FCS $(\%, v / v)$ & 12.00 & - & - \\
\hline Penicillin G & 0.17 & & - \\
\hline Streptomycin & 0.07 & & - \\
\hline Kanamycin sulphate & & 0.17 & - \\
\hline
\end{tabular}

aPartial listing of components of TCM-199 (Sigma, cat. no. M-5017). bFraction $\mathrm{V}$ (Sigma, cat. no. A-9647).

BSA: bovine serum albumin; PVA: polyvinyl alcohol; FCS: fetal calf serum; TALP: Tyrode's albumin lactate pyruvate; TBM: Tris-buffered medium.

(Table 1) and then washed gently until cumulus cells were removed with a $200 \mu \mathrm{l}$ automatic pipette. The IVF media used were modified TCM-199 (Coy et al., 1999), modified TALP (Rath et al., 1999) or modified TBM (Berger and Horton, 1988) and all were used after the previous protocols of preparation and equilibration overnight, including the mineral oil. Oocytes (groups of 50) were then cultured in $50 \mu \mathrm{l}$ microdrops covered with mineral oil and equilibrated for $30 \mathrm{~min}$ before introducing the spermatozoa.

Straws containing ejaculated frozen semen samples were thawed $\left(12 \mathrm{~s}\right.$ at $\left.50^{\circ} \mathrm{C}\right)$ and diluted in $10 \mathrm{ml}$ Beltsville thawing solution (BTS; Pursel and Johnson, 1975) at $37^{\circ} \mathrm{C}$. The pellet was washed at $150 \mathrm{~g}$ for $10 \mathrm{~min}$, placed on a 45/90 Percoll ${ }^{\circledR}$ (Pharmacia, Uppsala) gradient and centrifuged again at $500 \mathrm{~g}$ for $30 \mathrm{~min}$. The pellet was resuspended in the corresponding IVF medium $(5 \mathrm{ml})$ and washed again to remove the Percoll ${ }^{\circledR}$ at $150 \mathrm{~g}$ for $10 \mathrm{~min}$. Fifty microlitres of diluted spermatozoa was added to the microdrops containing the oocytes, giving a final sperm concentration of $2 \times 10^{6}$ cells ml-1. The final oocyte:spermatozoa ratio was thus 1:4000 for each of the three media.

Sperm concentration was selected according to previous assays using the three IVF media and the same batch of straws. The criterion was to find a sperm concentration at which measurable penetrations could be observed in the three media. 
Oocytes were co-cultured with spermatozoa in the IVF media for $5 \mathrm{~h}$. They were then transferred to $500 \mu \mathrm{l}$ NCSU-23 medium (Macháty et al., 1998) and cultured for an additional 7 days to the blastocyst stage.

\section{Assessment of the acrosome reaction pattern}

Sperm samples prepared for IVF as described above were incubated in TCM-199, TALP or TBM at $38.5^{\circ} \mathrm{C}$ under $5 \% \mathrm{CO}_{2}$. At $30 \mathrm{~min}$ intervals (from 0 to $150 \mathrm{~min}$ ), live and dead spermatozoa and the proportion of acrosomereacted spermatozoa were determined using fluorescein isothiocyanate-labelled peanut agglutinin (FITC-PNA) and propridium iodine $(\mathrm{PI})$ under an epi-fluorescence microscope. A $100 \mu \mathrm{l}$ aliquot of the sperm suspension was supplemented with $5 \mu \mathrm{lPNA}\left(200 \mu \mathrm{g} \mathrm{ml}^{-1}\right)$ and $5 \mu \mathrm{PI}$ $\left(200 \mu \mathrm{g} \mathrm{m}^{-1}\right)$, maintained at $38^{\circ} \mathrm{C}$ for $5 \mathrm{~min}$ and finally fixed in $10 \mu$ l paraformaldehyde (1\% (v/v) in PBS).

Three subsamples (200 spermatozoa per sample) were examined under the epi-fluorescence microscope ( $\times 1000)$, and spermatozoa were classified into three categories according to FITC-PNA staining: (i) spermatozoa considered to be alive and acrosome intact as determined by a lack of FITC-PNA or PI staining; (ii) spermatozoa not stained with PI with acrosomal areas stained with FITC-PNA considered to be alive and acrosome-reacted; and (iii) spermatozoa stained with PI considered to have damaged membranes.

\section{Assessment of cortical granules}

Methods of cortical granule visualization were based on those described by Yoshida et al. (1993b), with a few modifications by Wang et al. (1997b). Groups of five oocytes were analysed immediately after the end of the maturation period (control), after incubation in TCM-199, TALP or TBM for 2.5 or $5.0 \mathrm{~h}$, or after IVF in the three media for 2.5 or $5.0 \mathrm{~h}$. All the oocytes were denuded by pipetting and were washed in PBS. Subsequently, the oocytes were fixed with $3.7 \%(\mathrm{w} / \mathrm{v})$ paraformaldehyde in PBS for $30 \mathrm{~min}$ at room temperature and washed three times in PBS for 5 min each time. This procedure was followed by treatment with $0.1 \%(\mathrm{v} / \mathrm{v})$ Triton-X100 in PBS for $5 \mathrm{~min}$ and washing twice in PBS for $5 \mathrm{~min}$. Oocytes were then incubated in $100 \mu \mathrm{g}$ FITC-PNA ml-1 in PBS for 30 min in the dark. After staining, the oocytes were washed three times in PBS, mounted on slides with a coverslip secured by two lines of vaseline and sealed with nail polish.

The slides were examined under a Leica DMRB microscope with a TCS NT confocal module equipped with a krypto-argon ion laser for excitation of fluorescein for cortical granules. Images obtained using a Leica PL APO UV $63 \times 1.32$ NA oil objective were recorded digitally on a magnetic optical disk. Two sections of each oocyte were examined. The largest optical section was referred to as the equator of the oocyte, and the top surface section was taken as the oocyte cortex.

Cortical granule density of each oocyte was estimated using image analyst software MIP4 (Consulting of Digital
Imaging SL, Microm). In the equatorial image (Fig. 1a), two concentric circles marked the space immediately surrounding the oocyte plasma membrane (Fig. 1b). This measurement was used to record the area fraction (cortical granule-labelled area divided by the entire marked area $\times 100$ ). In the cortical image (Fig. 1c), one circle around the cortex of the oocyte was drawn to isolate the cortex area (Fig. 1d) and the same measurement of area fraction was calculated. More than 1300 isolated fluorescent points were measured in different regions of several oocytes, where they did not appear as aggregates, to obtain the real measurement of a single-labelled point. The calculated area for each point was $0.094 \mu \mathrm{m}^{2}$. Only six of the 1300 points indicated an area $>0.094 \mu \mathrm{m}^{2}$, corresponding to two or three aggregated fluorescent points. As a result, the final number of cortical granules per $100 \mu \mathrm{m}^{2}$ of cortex or equator could be calculated as the labelled area in $100 \mu \mathrm{m}^{2}$ divided by 0.094 .

\section{Assessment of zona pellucida solubility}

At the end of the maturation period, groups of 10 oocytes were used for the experiment before (control) or after incubation in TCM-199, TALP or TBM for 2.5 or $5.0 \mathrm{~h}$, or after IVF in the three media for 2.5 or $5.0 \mathrm{~h}$. The oocytes were transferred into PBS, washed by pipetting to remove surrounding cumulus cells or attached spermatozoa for the IVF groups, and added into $100 \mu \mathrm{l}$ of $0.1 \%(\mathrm{w} / \mathrm{v})$ pronase solution in PBS (Kim et al., 1996). Zonae pellucidae were continuously observed for dissolution under an inverted microscope equipped with a warm plate at $37^{\circ} \mathrm{C}$. The dissolution time of the zona pellucida of each oocyte was registered as the time interval between placement of the samples in pronase solution and that when the zona was no longer visible at a magnification of $\times 200$.

\section{Assessment of sperm penetration, pronucleus formation and developmental capacity of embryos}

Sperm penetration was assessed $18 \mathrm{~h}$ after IVF. A sample of oocytes from each of the different experimental groups was fixed in acetic acid:alcohol (1:3) for $24 \mathrm{~h}$, stained with $1 \%(\mathrm{w} / \mathrm{v})$ lacmoid and examined for evidence of sperm penetration and pronucleus formation under a phase contrast microscope ( $\times 400$ magnification).

Forty-eight hours after insemination, cleavage of oocytes was evaluated under a stereomicroscope equipped with a warm plate at $38.5^{\circ} \mathrm{C}$ and a special chamber connected to a $5 \% \mathrm{CO}_{2}$ permanent supply tube. Two four-cell stage embryos were recorded as cleaved. Blastocysts were assessed on day 7 by observation of a clear blastocoel under the stereomicroscope. Nuclei were counted by fixing and staining with Hoechst 33342 (1\% (w/v) in PBS).

\section{Statistical analysis}

Four replicate experiments were performed in each case. After in vitro maturation (IVM), oocytes were subjected to 

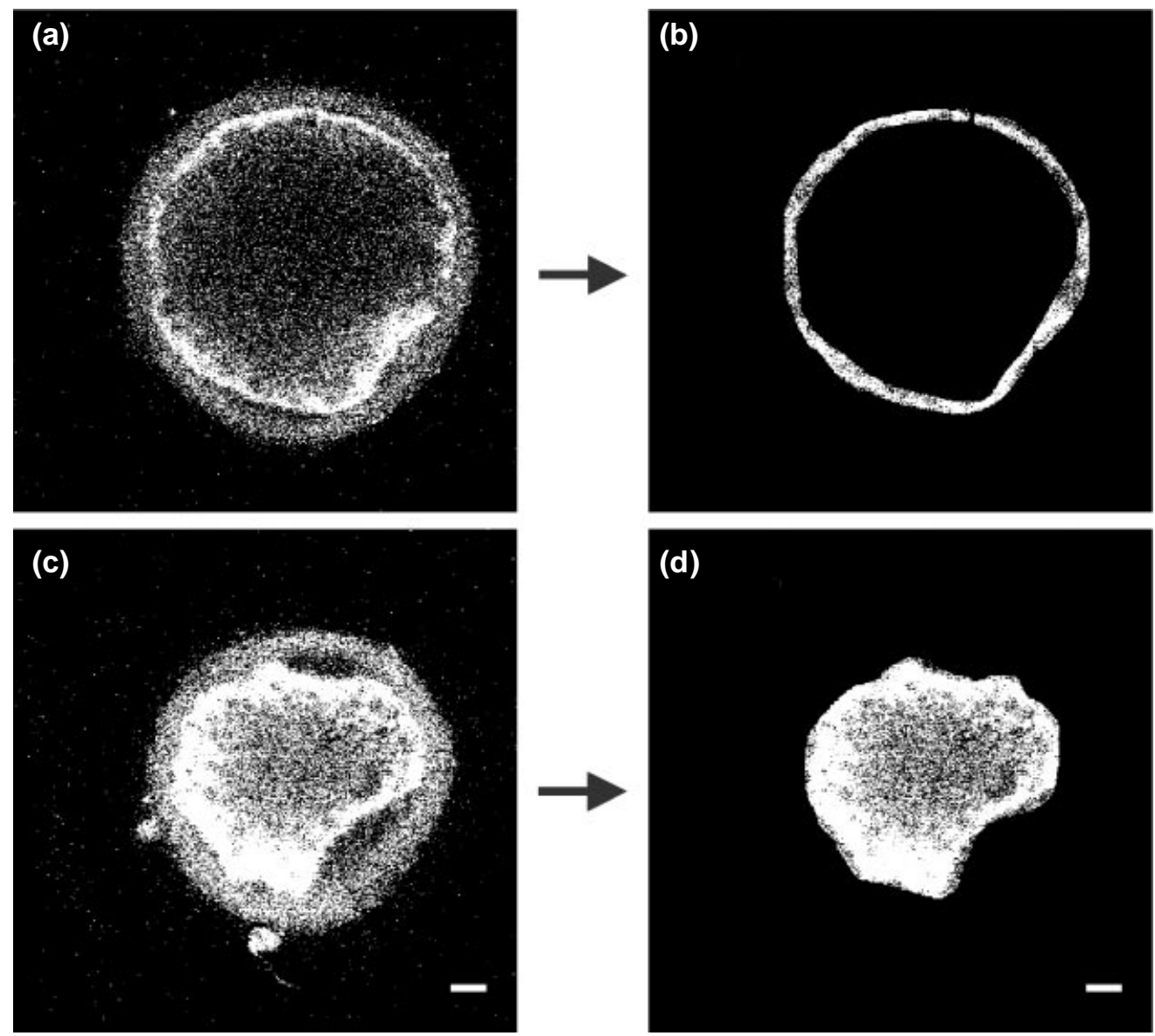

Fig. 1. Defining areas for cortical granule density assessment. (a) Equatorial image of a pig oocyte before isolating the area surrounding the plasma membrane. (b) Isolated equator. (c) Cortical image of the same oocyte before isolating the cortex area. (d) Isolated cortex. Scale bars represent $10 \mu \mathrm{m}$.

cortical granule density or ZP solubility assessment or to IVF. Data are presented as the mean \pm SEM and all percentages were modelled according to the binomial model of variables. The variables percentage of oocyte penetration, number of sperm cells per penetrated oocyte, male pronucleus formation, monospermy and performance, as well as percentages of cleavage and blastocyst formation, and number of cells per blastocyst were analysed by oneway ANOVA. Performance was defined as the percentage of monospermic oocytes with two pronuclei with respect to the total number of inseminated oocytes. Time of ZP dissolution, percentage of labelled area in the cortex and equator of the oocytes, and proportion of live acrosomereacted or acrosome-intact spermatozoa were analysed by two-way ANOVA considering the IVF medium and incubation time as main effects. In cortical granule and zona pellucida studies, the two categories of data (with and without spermatozoa) were analysed together and separately. When ANOVA revealed a significant effect, values were compared by the Tukey's test. A $P$ value of $<0.05$ was taken as statistically significant.

\section{Results}

\section{Acrosome reaction pattern}

The number of live acrosome-intact and acrosomereacted spermatozoa was dependent on the IVF medium $(P<0.001)$. The number of live spermatozoa with an intact acrosome was higher in spermatozoa cultured in TCM-199 than in spermatozoa cultured in TBM or TALP for 0-150 min (Fig. 2a). The number of acrosome-reacted spermatozoa cultured in TBM was higher than that recorded for TALP, which was in turn higher than that for TCM-199 $(P<0.001$, Fig. 2 b). The proportion of acrosome-reacted spermatozoa increased steadily during the first 30-60 min of culture in the three media.

\section{Cortical granule density and distribution}

Area fractions (AF) for the cortex and equator regions in the control group were $27.9 \pm 3.1$ and $7.6 \pm 0.9$, respectively. The IVF medium was found to affect the AF of each region of the oocyte significantly in the absence 
(a)

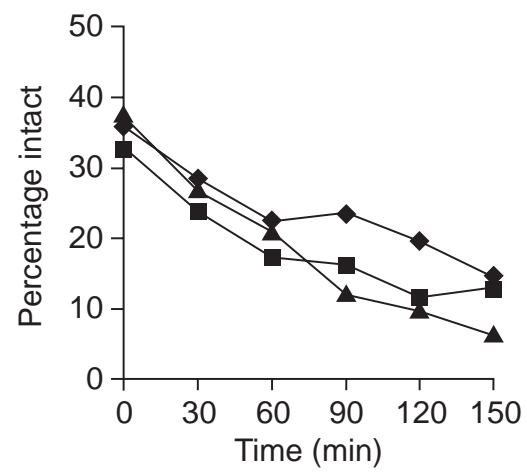

(b)

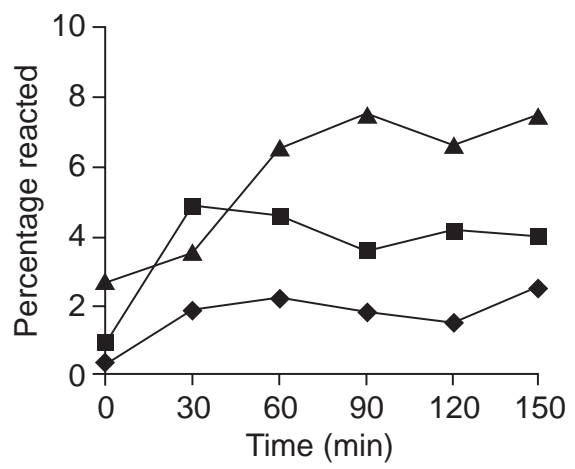

Fig. 2. Percentage of (a) acrosome-intact and (b) acrosome-reacted live pig spermatozoa during incubation in TCM-199 $(\downarrow)$, Tyrode's albumin lactate pyruvate (TALP) (ם) or Trisbuffered medium (TBM) ( $\mathbf{\Delta})$ for $150 \mathrm{~min}$ as assessed by fluorescein isothiocyanate-labelled peanut agglutinin (FITC-PNA) and propidium iodide (PI) staining.

(a)

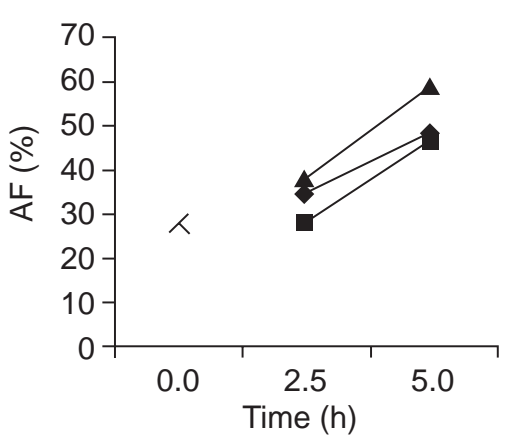

(c)

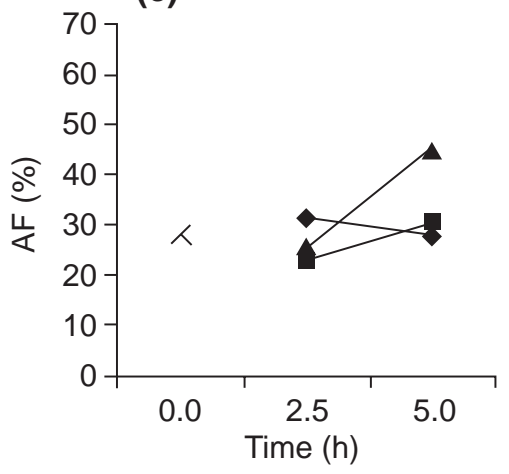

(b)

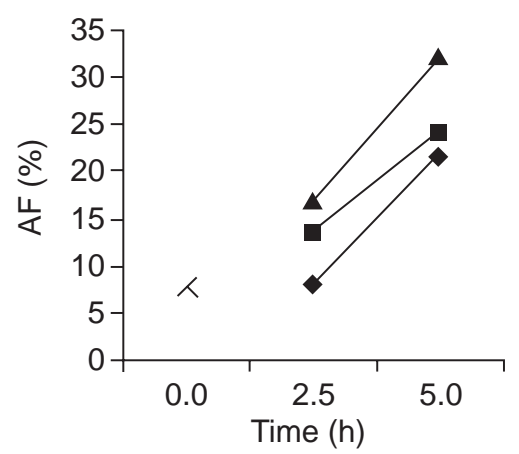

(d)

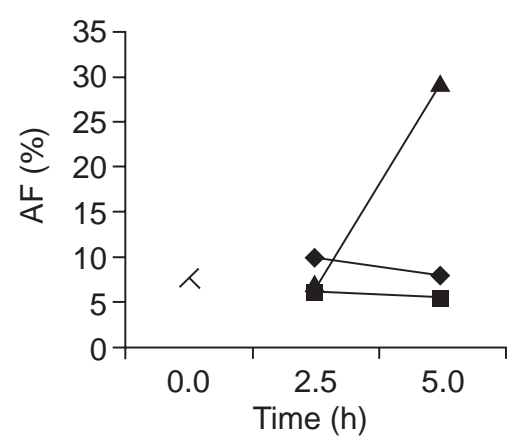

Fig. 3. Changes in cortical granule density (area fraction, $A F)$ in $(\mathrm{a}, \mathrm{c})$ the cortex and (b,d) equator of groups of 20 pig oocytes (in four replicates) incubated for 2.5 and $5.0 \mathrm{~h}$ in TCM-199 $(\bullet)$, Tyrode's albumin lactate pyruvate (TALP) ( $\square$ ) or Tris-buffered medium $($ TBM) $(\mathbf{A})$ in the $(a, b)$ absence or $(c, d)$ presence of spermatozoa.

(cortex, $P=0.026$; equator, $P=0.002$ ) and presence of spermatozoa (cortex, $P=0.011$; equator $P<0.001$; Fig. 3 ). TBM showed the highest effect and was associated with larger AF values than TCM-199 and TALP. In the absence of spermatozoa, incubation time also affected the distribution of cortical granules in the regions examined (Fig. 3a,b); the cortex and equator showed a notable increase in AF at $5 \mathrm{~h}$ $(P<0.001)$. The distribution pattern of cortical granules per region was similar in each media group; a higher density was recorded in the cortex than in the equator. 
Table 2. Changes in resistance of zona pellucida to pronase digestion in pig oocytes incubated in TCM199, TALP or TBM for 2.5 and $5.0 \mathrm{~h}$ in the presence or absence of spermatozoa

\begin{tabular}{|c|c|c|c|c|}
\hline & \multicolumn{4}{|c|}{ Zona pellucida resistance to pronase digestion (s) } \\
\hline & \multicolumn{2}{|c|}{ Without spermatozoa } & \multicolumn{2}{|c|}{ With spermatozoa } \\
\hline & $2.5 \mathrm{~h}$ & $5.0 \mathrm{~h}$ & $2.5 \mathrm{~h}$ & $5.0 \mathrm{~h}$ \\
\hline TCM-199 & $301.4 \pm 31.4^{\mathrm{a}}$ & $407.7 \pm 35.5^{\mathrm{a}}$ & $164.2 \pm 12.3$ & $151.6 \pm 16.1$ \\
\hline TALP & $203.1 \pm 13.7^{b}$ & $235.4 \pm 18.2^{b}$ & $136.9 \pm 8.8$ & $174.9 \pm 13.2$ \\
\hline TBM & $216.2 \pm 23.6^{\mathrm{ab}}$ & $189.1 \pm 16.8^{b}$ & $161.5 \pm 13.3$ & $148.3 \pm 11.4$ \\
\hline \multicolumn{5}{|c|}{ Source of variability ( $P$ value) } \\
\hline Medium & \multicolumn{2}{|c|}{$<0.001$} & \multicolumn{2}{|c|}{0.971} \\
\hline Time & \multicolumn{2}{|c|}{0.065} & \multicolumn{2}{|c|}{0.697} \\
\hline Medium $\times$ time & \multicolumn{2}{|c|}{0.028} & \multicolumn{2}{|c|}{0.074} \\
\hline
\end{tabular}

${ }^{\mathrm{a}-\mathrm{c}}$ Different superscripts in the same group indicate significantly different values $(P<0.05)$.

Control: $451.4 \pm 25.9 \mathrm{~s}$.

Data from four replicates.

TALP: Tyrode's albumin lactate pyruvate; TBM: Tris-buffered medium.

Table 3. Effect of the IVF medium (TCM-199, TALP or TBM) on fertilization variables in pigs

\begin{tabular}{lcccccc}
\hline Medium & $n$ & $\begin{array}{c}\text { Penetration } \\
(\%)\end{array}$ & $\begin{array}{c}\text { Number of spermatozoa } \\
\text { per oocyte }\end{array}$ & $\begin{array}{c}\text { Monospermy } \\
(\%)\end{array}$ & $\begin{array}{c}\text { Male pronucleus } \\
(\%)\end{array}$ & $\begin{array}{c}\text { Performance } \\
(\%)\end{array}$ \\
\hline TCM-199 & 92 & $59.8 \pm 5.1^{\mathrm{a}}$ & $1.4 \pm 0.1^{\mathrm{a}}$ & $65.4 \pm 6.5^{\mathrm{a}}$ & 100 & $39.1 \pm 5.1^{\mathrm{a}}$ \\
TALP & 111 & $72.9 \pm 4.2^{\mathrm{a}}$ & $2.8 \pm 0.3^{\mathrm{b}}$ & $40.7 \pm 5.5^{\mathrm{b}}$ & $97.5 \pm 1.7$ & $27.9 \pm 4.3^{\mathrm{ab}}$ \\
TBM & 103 & $19.4 \pm 3.9^{\mathrm{b}}$ & $1.2 \pm 0.1^{\mathrm{a}}$ & $80.0 \pm 9.2^{\mathrm{a}}$ & 100 & $15.5 \pm 3.6^{\mathrm{b}}$ \\
$P$ & & $<0.001$ & $<0.001$ & $<0.001$ & 0.396 & $<0.001$ \\
\hline
\end{tabular}

abValues in the same column with different superscripts are significantly different $(P<0.05)$.

Performance: percentage of penetrated monospermic oocytes with respect to the total number of oocytes.

Data from four replicates.

TALP: Tyrode's albumin lactate pyruvate; TBM: Tris-buffered medium.

When spermatozoa were introduced into the fertilization medium (Fig. 3c,d), the density of cortical granules per region was similar for the three media and the two incubation times, except for the TBM group at $5 \mathrm{~h}$, in which a higher $\mathrm{AF}$ was noted $(P<0.001)$ than in the other groups and control group $(P<0.005)$.

In general, cortical density with respect to the control group and the corresponding culture without spermatozoa decreased at $5 \mathrm{~h}$ when the spermatozoa were introduced in the culture medium, except for the TBM group in which no differences were found.

\section{Zona pellucida solubility}

The ZP of the oocytes matured in vitro took $451.4 \pm 25.9 \mathrm{~s}$ to dissolve when placed in pronase solution just after the maturation period (control group). For the incubated oocytes, the medium affected ZP resistance to pronase digestion (Table 2); the ZP of oocytes incubated in TCM199 was harder than that of oocytes cultured in TALP or TBM $(P<0.001)$. Resistance of the ZP tended to increase with incubation time $(P=0.065)$ after $5 \mathrm{~h}$, particularly in the TCM-199 group, with a significant interaction between medium and time $(P=0.028)$. In the presence of spermatozoa, no differences were noted according to the medium or time of culture. The results from the present study indicate that spermatozoa affect ZP solubility, and shorter dissolution times were recorded in the presence $(P<0.001)$ rather than in the absence of spermatozoa.

\section{In vitro fertilization and embryo development}

The percentage of metaphase II oocytes was $>95 \%$ in all the trials. The IVF medium affected all the variables evaluated $(P<0.001)$ except the percentage of male pronucleus formation, which was $>95 \%$ in all cases (Table 3). The percentage penetration was higher in TCM-199 $(59.8 \pm 5.1)$ or TALP $(72.9 \pm 4.2)$ than in TBM $(19.4 \pm 3.9)$. Although the percentage of monospermy was $80 \%$ in TBM, the final performance measured as the percentage of penetrated, monospermic oocytes with respect to the total number of oocytes was higher in TCM-199 (39.1 \pm 5.1$)$ than in TBM $(15.5 \pm 3.6)$ as a result of a lower percentage of penetration in TBM. 
Table 4. Effect of the IVF medium on the developmental capacity of pig oocytes

\begin{tabular}{lcccc}
\hline Medium & $n$ & Cleavage $(\%)$ & Blastocyst $(\%)^{*}$ & Number of cells per blastocyst \\
\hline TCM-199 & 218 & $41.74 \pm 3.3^{\mathrm{a}}$ & $7.69 \pm 2.8$ & $21.7 \pm 7.7$ \\
TALP & 198 & $38.38 \pm 3.4^{\mathrm{a}}$ & $7.89 \pm 3.1$ & $31.0 \pm 6.9$ \\
TBM & 198 & $26.26 \pm 3.1^{\mathrm{b}}$ & $3.85 \pm 2.7$ & $20.5 \pm 8.5$ \\
$P$ & & $<0.003$ & 0.620 & 0.649 \\
\hline
\end{tabular}

abValues in the same column with different superscripts are significantly different $(P<0.05)$.

*Percentage of blastocysts correlated with the number of cleaved oocytes.

Data from four replicates.

TALP: Tyrode's albumin lactate pyruvate; TBM: Tris-buffered medium.

For embryo development, the percentage cleavage was lower in TBM $(26.3 \pm 3.1)$ than in TCM-199 (41.7 \pm 3.3$)$ or TALP $(38.4 \pm 3.5 ; P<0.003)$. However, the percentage of blastocysts formed was low in all three media (ranging from $7.89 \pm 3.1$ to $3.85 \pm 2.7$ ), as was their quality determined as the number of Hoechst-stained nuclei (Table 4).

\section{Discussion}

Under physiological conditions, the acrosome reaction starts immediately after primary binding of a sperm cell to the ZP (Wassarman, 1992). Pre-acrosome-reacted sperm cells are considered to be unable to fertilize the oocyte. In vitro, a spontaneous acrosome-reaction can be induced by different reagents (Funahashi and Nagai, 2001). Furthermore, a large number of capacitated or partially acrosome-reacted spermatozoa surrounding oocytes matured in vitro may be among the main causes of increased percentages of penetration and polyspermy (Funahashi and Nagai, 2001). In addition, it has been demonstrated that a higher proportion of spermatozoa are capacitated when frozen-thawed semen samples are used compared with fresh semen samples (Maxwell and Johnson, 1997). In some reports, the acrosome reaction is considered as an indicator of sperm capacitation (Flesch and Gadella, 2000), as this event takes place in capacitated cells. With these premises, a suitable IVF medium for frozen-thawed spermatozoa should not give rise to a premature acrosome reaction and, at the same time, should allow small populations of capacitated but not fully reacted sperm cells to slowly approach the ZP of the oocyte during culture. Results from the present study show that the proportion of acrosomereacted spermatozoa increased steadily during culture in the three IVF media used, but was always higher for TBM. Therefore, it might be expected that culture in the TBM would lead to highest penetration and polyspermy percentages, but this was not the case under our experimental conditions. On the contrary, penetration was under $20 \%$ and monospermy was $80 \%$. A possible interpretation of these results is that, under the present IVF conditions, the number of capacitated, non-fully-reacted spermatozoa reaching the oocyte may have been low in the TBM, as a result of poor motility and a premature acrosome reaction in a large proportion of the live spermatozoa. In a previous study, spermatozoa resuspended immediately in TBM after Percoll treatment showed considerable decrease in motility compared with those placed in TCM-199 or TALP medium (P. Coy, J. Gadea, R. Romar and C. Matás, unpublished). Experimental evidence indicates that spermatozoa with poor motility, even if they are able to undergo the acrosome reaction, are unlikely to penetrate the surrounding layers of cumulus oophorus cells and the $\mathrm{ZP}$, and that decreased motility is the best predictor of fertilization failure (Berger et al., 1996; Rhemrev et al., 2001). If it is assumed that spermatozoa begin to penetrate oocytes $2-3 \mathrm{~h}$ after insemination and that the percentage fertilization increases for up to $22 \mathrm{~h}$ thereafter (Wang et al., 1998a; Marchal et al., 2002), it is possible that, under our experimental conditions, the proportion of spermatozoa ready for penetration at the precise time in TBM was insufficient. In addition, the large proportion of fully acrosome-reacted cells and delayed hyperactivation would give rise to a lower percentage of penetration. Alternatively, IVF results may be more dependent on oocyte quality than on sperm survival and capacitation, as suggested by Marchal et al., 2002) and perhaps it is the cortical reaction pattern of the oocyte that is responsible for low penetrability. This possibility is discussed below.

Changes in the distribution of cortical granules in pig oocytes were first demonstrated by electron microscopy (Cran and Cheng, 1985, 1986). This study was followed by the development of a relatively simple detection system based on investigations in mice (Ducibella et al., 1988) by Yoshida et al. (1993b), involving the use of confocal laser microscopy following treatment with fluorescently labelled lectins. From these studies, it may be deduced that cortical granules inside the pig oocyte can be visualized using FITCPNA when oocytes have been previously treated with Triton-X100 as a permeabilizing substance.

After analysing the first samples, it was observed that the concentration of $100 \mu \mathrm{g}$ FITC-PNA ml-1 was too high to produce realistic measurements in absolute values, as labelling was noted in the zona pellucida. As reported by Yoshida et al. (1993b) and confirmed by the lectin-gold technique and immunocytochemistry on transmission electron microscopy studies ( $M$. Avilés and P. Coy, unpublished), the porcine ZP is negative to PNA. As ZP labelling in our images could not be attributed to the 
background because the surface immediately around the oocyte appeared to be clean and results for control groups at 50 and $20 \mu \mathrm{g}$ FITC-PNA ml-1 were the same as that of the control group described in the present study, we decided to use $100 \mu \mathrm{g}$ FITC-PNA ml-1 so that the results would be comparable with those reported in papers using this technique in pigs (Yoshida et al., 1993b; Kim et al., 1996; Wang et al., 1997b,c, 1998b). As a consequence, although the differences among groups are valid, data were not expressed as the number of cortical granules per $100 \mu \mathrm{m}^{2}$ because, in our opinion, the procedure is unable to offer realistic absolute values of this parameter and it is not realistic to attribute every fluorescent point to one cortical granule.

In the cortex and equator regions, an increasing cortical granule density was observed within the culture time in the absence of spermatozoa and stable density in the presence of spermatozoa in the TCM-199 and TALP media. However, for oocytes in TBM, cortical granule density increased from 2.5 to $5.0 \mathrm{~h}$ regardless of the presence or absence of spermatozoa. A physiological interpretation of this finding was proposed. In vivo, cortical granules increase in number during the late stages of oocyte maturation. Up to $20 \mathrm{~h}$ after hCG treatment, $<20 \%$ of granules may be detected within $4 \mathrm{~nm}$ of the plasma membrane. From 30 to $40 \mathrm{~h}$, this value increases to $75 \%$ and by $50 \mathrm{~h}(8-10 \mathrm{~h}$ after ovulation) virtually $100 \%$ have migrated to form a monolayer (Cran and Cheng, 1985). Numerous Golgi elements have been described as prominent and actively involved in cortical granule formation from the time of ovulation to sperm penetration (Guraya, 1985). Moreover, in some species, such as rabbits, the continuous formation of cortical granules in oviductal oocytes has been demonstrated (Guraya, 1985). In the present study, after $44 \mathrm{~h}$ of in vitro maturation, the oocytes were introduced into the IVF medium for a further $5 \mathrm{~h}$. This corresponds to $49 \mathrm{~h}$ from the 'artificial' LH surge. Thus, the observed increase in cortical granule density is consistent with the situation in vivo. The presence of spermatozoa in TCM-199 and TALP media halts the mean increase in cortical granule density but this is not the case in TBM. This finding is consistent with the penetration results, which were higher in TCM-199 and TALP than in TBM, indicating that contact between oocytes and spermatozoa in TCM-199 and TALP prevents more cortical granules appearing or more probably involves oocyte activation and beginning of cortical granule exocytosis. In TBM, this would occur only in a few oocytes ( $20 \%$ of those penetrated), giving a mean cortical granule density closer to that of the incubated oocytes in the absence of spermatozoa. This finding would be in agreement with the findings discussed above, that is, a premature acrosome reaction and poor motility of spermatozoa resuspended in this medium resulting in a low penetration percentage under IVF conditions used in the present study.

Sperm-egg interaction may involve receptor- or fusionmediated stimulation of different molecules and secondary messengers, such as inositol 1,4,5-trisphosphate $\left(\mathrm{IP}_{3}\right)$ and diacylglycerol (DAG) (Ducibella, 1998), leading to increased intracellular $\mathrm{Ca}^{2+}$ and cortical granule secretion in the oocyte. But how long does the cortical reaction take? In sea urchins, cortical granules undergo exocytosis within seconds (Abbottt and Ducibella, 2001). In hamsters, cortical granule exocytosis takes 9 min to complete (Stewart-Savage and Bavister, 1991) and in mouse eggs, fluorescence microscopy indicates that exocytosis starts within 5-10 min and continues for up to $1 \mathrm{~h}$ (Tahara et al., 1996). Results from the present study in pigs show that $5 \mathrm{~h}$ after IVF, there is only discrete evidence of a cortical reaction, as even in TCM-199 and TALP, a large proportion of cortical granules remained in the oocyte compared with the control. Other authors have reported that in a TCM-199 in vitro maturation/IVF system in pigs, 58\% of the oocytes showed no signs of cortical granule exocytosis, 33\% showed some degree and only $8 \%$ of the oocytes showed complete exocytosis $6 \mathrm{~h}$ after insemination (Wang et al., 1997c). These results are consistent with the results from the present study, although in the present study, the use of fetal calf serum cannot explain this delayed cortical reaction (as suggested by these authors) because it is not included in the TALP or TBM composition. In addition, further findings by Wang et al. (1998b) indicated an intense cortical reaction in oocytes $6 \mathrm{~h}$ after IVF in serum-free TBM, similar to that produced in oocytes matured in vivo. This finding could indicate a slower cortical reaction under the conditions used in the present study in the same medium.

The results discussed above indicate that there is a slow cortical reaction in in vitro systems. However, the present study is one of very few investigations designed to evaluate cortical granule density close to the time of fertilization. It is shown here that no intense cortical reaction takes place after $5 \mathrm{~h}$ of contact with spermatozoa, given the fact that the AF was no lower than control values, at least in the TCM199 and TALP groups. However, a response to the spermatozoon was shown in both these media, or the sustained increase in the number of cortical granules observed in the absence of sperm cells was interrupted when spermatozoa were introduced in the incubation medium. As a consequence, the cortical reaction may be much slower than in vivo and may possibly be related to the lack of response to ZP hardening after fertilization.

It has been established that the ZP of oocytes matured in vivo is highly resistant to pronase digestion (Wang et al., $1998 b)$. This hardening may be the result of deposition of oviductal secretions. In vitro, the preculture of oocytes in the presence of $30 \%$ oviductal fluid increases the resistance of the ZP to digestion by pronase (Kim et al., 1996). In the present study, the ZP of control oocytes subjected to $44 \mathrm{~h}$ of IVM took $451.4 \pm 25.9 \mathrm{~s}$ to be digested, indicating that the ZP of our IVM oocytes was not resistant to pronase digestion. Even in this situation, oocytes incubated in TCM199 showed the higher resistance to ZP dissolution after culturing in absence of spermatozoa. This finding could be related to the higher percentage of monospermy observed 
after IVF compared with that noted for the TALP medium. Nonetheless, none of the three IVF media showed a ZP hardening effect comparable with that produced by oviductal fluid, which is in agreement with results from other studies.

Recent findings provide strong support for a difference in the morphology of the $\mathrm{ZP}$ and the $\mathrm{ZP}$ reaction upon sperm penetration between in vitro-matured and ovulated oocytes (Funahashi et al., 2000). In the present study, there were no significant differences in ZP hardening at the time of sperm penetration in the three IVF media, and the dissolution times ranged between $136.9 \pm 8.8 \mathrm{~s}$ and $174.9 \pm 13.2 \mathrm{~s}$. This finding would corroborate the fact that the changes observed in the ovulated oocytes in terms of hardening did not occur in vitro independently of the IVF medium used. However, in contrast, there was evident softening of oocytes matured in vitro used in the present study in the presence of spermatozoa compared with the same groups in the absence of spermatozoa. This finding could be due to the effect of the acrosome enzymes from the reacted spermatozoa, which could modify the ZP, rendering it less resistant to pronase. In rat oocytes, the ZP hardening is a protease-dependent event (Wassarman, 1988) and during the acrosome reaction certain proteases such as acrosine are released (Yanagimachi, 1994). Such enzymes could be responsible for molecular and structural changes in the ZP of pig oocytes, as has been suggested for other species (Hedrick and Wardrip, 1987). From the results of the present study, with the sperm concentration used, it can be inferred that the number of reacted spermatozoa around the oocyte is probably several fold higher than the number found under the in vivo condition, and this could explain the same ZP softening observed in the three groups from the first $2.5 \mathrm{~h}$ of culture.

The results from the present study highlight how far we still are from achieving biological properties of gametes close to those observed in the physiological setting. Neither the cortical reaction nor ZP hardening correspond to observations in vivo, and the acrosome reaction pattern depends on the IVF medium used. Thus, it is not surprising that significant differences were observed in the percentages of penetration and monospermy depending on the medium used, and confirmed once again that, under our IVF conditions, TCM-199 and TALP media gave rise to higher penetration percentages in comparison with TBM. In addition, the use of TCM-199 and TBM was related to higher percentages of monospermy than was use of TALP. These results gave rise to cleavage percentages that always exceeded the performance percentage, as this variable includes all morphologically normal two- to four-cell stage embryos detected by microscopy. In our opinion, this criterion is not the best to evaluate the success of the system; the number of cells per blastocyst is thought to be more appropriate. In the present study, this corresponded to a lower value than expected with respect to blastocysts produced in vivo, as reported by Wang et al. (1999). Even knowing that these variables are dependent on the sperm concentration, this type of question needs to be addressed to improve IVF procedures.

In conclusion, the acrosome reaction depends on the IVF medium. The confocal study of cortical granules is not an adequate system to obtain absolute values in terms of the number of cortical granules per area studied, but is useful for comparing density changes during culture. Under IVF conditions in the present study, cortical granules increase during culture in the absence of spermatozoa; the cortical reaction starts but it is not complete after $5 \mathrm{~h}$ of oocytespermatozoon contact; ZP hardening does not occur but a marked softening is observed in presence of spermatozoa; and finally, the blastocyst percentage, which is currently the most realistic measure of the effectiveness of an IVF system, is still very low. Thus, given the present low output in the in vitro production of pig embryos, there is an urgent need to develop an IVF medium and environment that closely resemble physiological conditions.

This research was supported by grant AGL 2000-0485-C02-01. The authors thank Luz Campos, Teresa Castell and Manuel Avilés for technical help with confocal microscopy, image analysis and electron microscopy, respectively.

\section{References}

Abbott AL and Ducibella T (2001) Calcium and the control of mammalian cortical granule exocytosis Frontiers in Bioscience 6 792-806

Abeydeera LR, Wang WH, Cantley TC, Rieke A and Day BN (1998) Coculture with follicular shell pieces can enhance the developmental competence of pig oocytes after in vitro fertilization: relevance to intracellular glutathione Biology of Reproduction 58 213-218

Berger T and Horton MB (1988) Evaluation of assay conditions for the zonafree hamster ova bioassay of boar sperm fertility Gamete Research 19 101-111

Berger T, Anderson DL and Penedo MCT (1996) Porcine sperm fertilizing potential in relationship to sperm functional capacities Animal Reproduction Science 44 231-239

Cheng WTK (1985) In Vitro Fertilization of Farm Animal Oocytes PhD Thesis, University of Cambridge

Coy $\mathbf{P}$ and Romar $\mathbf{R}$ In vitro production of pig embryos. A point of view Reproduction, Fertility and Development (in press)

Coy P, Ruiz S, Romar R, Campos I and Gadea J (1999) Maturation, fertilization and complete development of porcine oocytes matured under different systems Theriogenology 51 799-812

Cran DG and Cheng WTK (1985) Changes in cortical granules during porcine oocyte maturation Gamete Research 11 311-319

Cran DG and Cheng WTK (1986) The cortical reaction in pig oocytes during in vivo and in vitro fertilization Gamete Research 13 241-251

Ducibella T (1998) Biochemical and cellular insights into the temporal window of normal fertilization Theriogenology 49 53-65

Ducibella T, Anderson E, Albertini DF, Aalberg J and Rangarajan S (1988) Quantitative studies of changes in cortical granule number and distribution in the mouse oocyte during meiotic maturation Developmental Biology $130184-197$

Fissore RA, Dobrinsky JR, Balise JJ, Duby RT and Robl JM (1992) Patterns of intracellular $\mathrm{Ca}^{2+}$ concentrations in fertilized bovine eggs Biology of Reproduction 47 960-969

Flesch FM and Gadella BM (2000) Dynamics of the mammalian sperm plasma membrane in the process of fertilization Biochimica et Biophysica Acta 1469 197-235

Funahashi H and Day BN (1993) Effects of the duration of exposure to supplemental hormones on cytoplasmic maturation of pig oocytes in vitro. Journal of Reproduction and Fertility 98 179-185 
Funahashi H and Nagai T (2001) Regulation of in vitro penetration of frozen-thawed boar spermatozoa by caffeine and adenosine Molecular Reproduction and Development 58 424-431

Funahashi H, Ekwall H and Rodriguez-Martinez H (2000) Zona reaction in porcine oocytes fertilized in vivo and in vitro as seen with scanning electron microscopy Biology of Reproduction 63 1437-1442

Guraya SS (1985) Biology of Ovarian Follicles in Mammals pp 27-34. Springer Verlag, New York

Hedrick JL and Wardrip NJ (1987) On the macromolecular composition of the zona pellucida from porcine oocytes Developmental Biology 121 478-488

Kim NH, Funahashi H, Abeydeera LR, Moon SJ, Prather RS and Day BN (1996) Effects of oviductal fluid on sperm penetration and cortical granule exocytosis during fertilization of pig oocytes in vitro. Journal of Reproduction and Fertility 107 79-86

Macháty Z, Day BN and Prather RS (1998) Development of early porcine embryos in vitro and in vivo. Biology of Reproduction 59 451-455

Marchal R, Peláez J, Terqui M and Mermillod P (2002) Effect of sperm survival and CTC staining pattern on in vitro fertilization of porcine oocytes Theriogenology 57 1917-1927

Mattioli M, Bacci ML, Galeati G and Seren E (1989) Developmental competence of pig oocytes matured and fertilized in vitro. Theriogenology 31 1201-1207

Maxwell WM and Johnson LA (1997) Chlortetracycline analysis of boar spermatozoa after incubation, flow cytometric sorting, cooling, or cryopreservation Molecular Reproduction and Development $\mathbf{4 6}$ 408-418

Petters RM and Wells KD (1993) Culture of pig embryos Journal of Reproduction and Fertility Supplement 48 61-73

Pursel VG and Johnson LA (1975) Freezing of boar spermatozoa; fertilizing capacity with concentrated semen and a new thawing procedure Journal of Animal Science 40 99-102

Rath D, Long CR, Dobrinsky JR, Welch GR, Schreier LL and Johnson LA (1999) In vitro production of sexed embryos for gender preselection: high-speed sorting of X-chromosome bearing sperm to produce pigs after embryo transfer Journal of Animal Science 77 3346-3352

Rhemrev JPT, Menkveld R, Roseboom TJ, Overled FWPC, Teerlink T, Lombard C and Vermeiden PW (2001) The acrosome index, radical buffer capacity and number of isolated progressively motile spermatozoa predict IVF results Human Reproduction 16 1885-1892

Stewart-Savage J and Bavister BD (1991) Time course and pattern of cortical granule breakdown in hamster eggs after sperm fusion Molecular Reproduction and Development 30 390-395

Tahara M, Tasaka K, Masumoto N, Mammoto A, Ikebuchi Y and Miyake A
(1996) Dynamics of cortical granule exocytosis at fertilization in living mouse eggs American Journal of Physiology 270 1354-1361

Wang WH, Sun QY, Hosoe M, Shioya Y and Day BN (1997a) Quantified analysis of cortical granule distribution and exocytosis of porcine oocytes during meiotic maturation and activation Biology of Reproduction 56 1376-1382

Wang WH, Abeydeera LR, Cantley TC and Day BN (1997b) Effects of oocyte maturation media on development of pig embryos produced by in vitro fertilization Journal of Reproduction and Fertility 111 101-108

Wang WH, Hosoe M and Shioya Y (1997c) Induction of cortical granule exocytosis of pig oocytes by spermatozoa during meiotic maturation Journal of Reproduction and Fertility 109 247-255

Wang WH, Macháty Z, Abeydeera L, Prather R and Day BN (1998a) Parthenogenetic activation of pig oocytes with calcium ionophore and the block to sperm penetration after activation Biology of Reproduction 58 1357-1366

Wang WH, Abeydeera LR, Prather RS and Day BN (1998b) Morphologic comparison of ovulated and in vitro-matured porcine oocytes, with particular reference to polyspermy after in vitro fertilization Molecular Reproduction and Development 49 308-316

Wang WH, Abeydeera LR, Han YM, Prather RS and Day BN (1999) Morphologic evaluation and actin filament distribution in porcine embryos produced in vitro and in vivo. Biology of Reproduction 60 1020-1028

Wassarman PM (1988) Zona pellucida glycoproteins Annual Review of Biochemistry 57 415-442

Wassarman PM (1992) Mouse gamete adhesion molecules Biology of Reproduction 46 186-191

Yanagimachi R (1994) Mammalian fertilization. In The Physiology of Reproduction pp 189-317 Eds E Knobil and JD Neill. Raven Press, New York

Yoshida M, Mizoguchi Y, Ishigaki K, Kojima T and Nagai T (1993a) Birth of piglets derived from in vitro fertilization of pig oocytes matured in vitro. Theriogenology 39 1303-1311

Yoshida M, Cran DG and Pursel VG (1993b) Confocal and fluorescence microscopic study using lectins of the distribution of cortical granules during the maturation and fertilization of pig oocytes Molecular Reproduction and Development 36 462-468

Received 22 February 2002.

First decision 27 March 2002.

Revised manuscript received 18 April 2002.

Accepted 8 May 2002. 